\title{
DEFICIENCY OF ADAMTS-13 IN SEPSIS PATIENTS AND ITS CORRELATION WITH MORBIDITY/ MORTALITY
}

\author{
Syed Owais Ali, Chaudhry Altaf Hussain, Dawood Ahmad, Rafia Mahmood, Syeda Sarwat Fatima, Taqdees Fatima* \\ Armed Forces Institute of Pathology/National University of Medical Sciences (NUMS) Rawalpindi Pakistan, *Combined Military \\ Hospital/National University of Medical Sciences (NUMS) Rawalpindi Pakistan
}

\begin{abstract}
Objective: To detect ADAMTS-13 deficiency in sepsis patients and its effect on in-hospital morbidity and mortality in adult patients of sepsis.

Study Design: Cross sectional study.

Place and Duration of Study: Armed Forces Institute of Pathology (AFIP) and Combined Military Hospital (CMH) Rawalpindi, from Apr 2017 to Apr 2018.

Methodology: All indoor patients admitted at Combined Military Hospital Rawalpindi and diagnosed as suffering from sepsis at Armed Forces Institute of Pathology according to the guidelines of the Critical Care Society of Medicine Consensus Conference Committee. Forty sepsis patients were recruited for the study. Two groups were formed on the basis of ADAMTS-13 antigen levels, non-deficient and deficient. Basic haematological and clinical parameters were recorded. Levels of ADAMTS-13 antigen were analyzed by using Technozyme kit byenzyme-linked immunosorbent assay (ELISA) and level less than $0.40 \mathrm{IU} / \mathrm{ml}$ was taken deficient as defined by manufacturer. Comparison was done between ADAMTS-13 deficient and non-deficient group with regard to clinical and haematological characteristics, ICU stay, hospital admission days and in-hospital mortality. Healthy controls of same age and gender were also observed for ADAMTS-13 antigen level.

Results: Among total 40 sepsis patients, thirty two (80\%) were males and eight $(20 \%)$ were females with a mean age of $61 \pm 15$ years (range: 30-85 years). ADAMTS-13 deficiency $(<0.40 \mathrm{IU} / \mathrm{ml})$ was found in $28(70 \%)$ of patients while $12(30 \%)$ had normal antigen levels. In patients with ADAMTS-13 deficiency, twenty four $(85.7 \%)$ had low platelet levels $<150 \times 10^{9} / 1$, mean hospital stay $19.2 \pm 7.6$ days and in-hospital mortality was seen in eight $(28.6 \%)$ patients, in comparison of non-deficient group in which platelet levels were equal or $>150 \times 10^{9} / 1$, mean hospital stay was $10.2 \pm 3.6$ days and in-hospital mortality was in one $(8.3 \%)$ patient respectively. ADAMTS-13 antigen level was found sufficient in healthy controls.

Conclusion: Majority of the sepsis patients were found ADAMTS-13 deficient. Deficiency exhibit long term hospital admission, thrombocytopenia and increase in hospital mortality. ADAMTS-13 deficiency might play a role in sepsis induced thrombocytopenia and in hospital mortality. More studies are recommended on the subject with larger sample size to evaluate its role in sepsis.
\end{abstract}

Keywords: A disintegrin and metalloprotease with a thrombospondin type1 motif, Member 13, Disseminated intravascular coagulation, Enzyme-linked immunosorbent assay, Thrombotic thrombocytopenic purpura.

\footnotetext{
This is an Open Access article distributed under the terms of the Creative Commons Attribution License (http://creativecommons.org/licenses/by/4.0), which permits unrestricted use, distribution, and reproduction in any medium, provided the original work is properly cited.
}

\section{INTRODUCTION}

A clinical syndrome "Sepsis" is characterized by systemic inflammatory response secondary to infection 1 . United States of America declared this $10^{\text {th }}$ leading cause of death2 ${ }^{2}$ Sepsiscaused mortality is more than total number of deaths by AIDS, prostate cancer and breast cancer combined ${ }^{3}$. Hospitalizations due to sepsis are more than

Correspondence: Dr Syed Owais Ali, Dept of Haematology, Armed Forces Institute of Pathology, Rawalpindi Pakistan Received: 26 Jun 2018; revised received: 11 Jul 2019; accepted: 29 Jul 2019 heart diseases and it has become a leading cause of hospital expenditure 4 . Ninety percentdoctors feel that sepsis is a significant financial burden on the health care system in their country ${ }^{5}$.

In pathophysiology of sepsis, endothelial dysfunction plays an important role. In healthy state, endothelial cells inhibit coagulation, prevent platelet aggregation, and control vascular tone and permeability ${ }^{4}$. Duringsepsis, endothelial activation induces a pro-coagulant condition that is associated with widespread microvascular 
thrombosis. Endothelium released von Willebr and factor (vWF) and mediates platelet adhesion and aggregation at sites of vascular injury. It is released from the stimulated endothelium to form the hyperactive and ultra large von Willebrand factor ${ }^{6}$.

Weibel-Palade bodies within the endothelial cells and alpha granules of platelets store $\mathrm{vWF}^{7}$. It is released from the stimulated endothelium as ultra large (UL) multimers. Thisinitial size of ULvWF depends upon ADAMTS-13 as it cleaves this and converts it into small active monomers ${ }^{8}$. Decrease cleavage of ULvWF due to reduced ADAMTS-13 antigen level or activity results in disseminated platelet-rich thrombi in the microcirculation and leads to sepsis, DIC, TTP and organ damage ${ }^{9}$. Many other conditions like cirrhosis, SLE, DIC and carcinomas also exhibit decrease level of this enzyme ${ }^{10}$. Majority of sepsis patients of all age groups died every year in Emergency departments of healthcare centers ${ }^{11}$. It has been observed in sepsis patients that decreased ADAMTS-13 level negatively correlates with the disease prognosis ${ }^{12}$. Based on this strong affiliation of ADAMTS-13 with sepsis, we conducted this research to detect ADAMTS-13 deficiency and its impact on in-hospital morbidity and mortality in adult patients of sepsis. We also observed its level in our healthy controls of same age and gender. Furthermore, we also correlated its deficiency with sepsis associated fall of platelets, stay in intensive care unit/ hospital, death in hospital in comparison of non-deficient group.

\section{METHODOLOGY}

This cross sectional descriptive study was conducted at Armed Forces Institute of Pathology (AFIP) and Combined Military Hospital (CMH) Rawalpindi, over a period of one year from 25 April 2017 to 24 April 2018, after the approval of institutional Ethics committee and CPSP. All patients/their attendants were completely guided about the study and use of data, only those who showed willingness and gave written permission were sampled. Forty adult patients age between $30-85$ years of both gender, admitted to intensive care unit (ICU) of Combined Military Hospital Rawalpindi, diagnosed to have sepsis were enrolled in study. Mean age was $61 \pm 15$ years (range: $30-85$ years). The sample size calculated was 40 by using the WHO calculator with a confidence level of $95 \%$, anticipated population proportion $(\mathrm{P})$ of .85 and absolute precision (d) of 10. Non probability consecutive sampling was done. Manufacturer's provided value for ADAMTS-13 deficiency $(<0.40 \mathrm{IU} / \mathrm{ml})$ was confirmed by performing tests on same number of age and gender matched healthy controls and no any deficiency was found in healthy control population. Sepsis cases included in the study were had positive blood culture, documented infection and signs of systemic inflammationas per the criteria of society of Critical Care Medicine Consensus Conference Committee. Selected patients met at least 3 out of the 4 systemic inflammatory response criteria ${ }^{13}$ :

Body core temperature more than $38.5^{\circ} \mathrm{C}$ (Hyperthermia) or less than $36^{\circ} \mathrm{C}$ (Hypothermia).

Tachycardia more than two standard deviation above normal for age.

Mean respiratory rate more than two standard deviation above normal for age.

Total leukocyte count increased or decreased for age in the presence of a known or conjecture infection.

Observation of pathogenic microorganisms through positive blood/ body fluid culture.

Conditions that might interfere with ADAMTS-13 level, like patients with known congenital heart disease, chronic lung disease, encephalitis, rheumatologic disease, thrombotic or bleeding disorder, pregnancy, third degree burn injury, TTP, ITP, HUS, and liver disease, or those who received blood transfusion within seven days were excluded from the study.

With aseptic technique, venous blood ( $3 \mathrm{ml})$ was taken from every patient in tubes containing $3.2 \%$ sodium citrate anticoagulant. Platelet poor plasma was obtained after centrifugation of 
samples. Specimens were labelled and stored at $80^{\circ} \mathrm{C}$ until analysis.

ADAMTS-13 antigen level was assessed by a commercially available chromogenic enzymelinked immunosorbent assay (ELISA) (TECHNOZYM® ADAMTS-13 activity, Technoclone, Vienna, Austria). All measurements/readings were taken on ELISA reader (BIORAD PR S100) at wave length of $450 \mathrm{~nm}$ and ADAMTS-13 activity $<0.40 \mathrm{IU} / \mathrm{ml}$ was considered deficient as defined by manufacturer. Impaired antigen level group was compared with patients who had adequate levels. Comparison was done with regard to age, gender, haematological characteristics and inhospital mortality between these two groups.

All relevant information was entered and interpreted using SPSS (Statistical Package for Social Sciences) version 24. A $p$-value less than 0.05 was considered statistically significant. Different Statistical tools including frequency, mean, median and Chi-square tests were used for comparison/significance of input depending on the scale of measurement.

\section{RESULTS}

Out of forty patients with sepsis admitted in the intensive care unit, thirty two (80\%) were males and eight $(20 \%)$ were females with a mean age of $61 \pm 15$ years (range: 30-85 years). On with ADAMTS-13 deficiency, twenty four (85.7\%) had platelets of $<150 \times 10^{9} / 1$, twenty three $(82.1 \%)$ patients had platelets $<100 \times 10^{9} / 1$ and fourteen $(50 \%)$ had platelets < $50 \times 10^{9} / 1$. Low ADAMTS-13 level were also associated with prolonged PT and aPTT. However, there was no statistically significant association between ADAMTS-13 levels and the total leukocyte count. Meanhospital stay was 19.2 ( \pm 7.6$)$ days with a mean of $9.6 \pm 2.5$ days in ICU which is significantly high in comparison of non-deficient group of $10.2 \pm 3.6$ and $5.5 \pm 1.6$ respectively. Twenty-two (78.6\%) patients required mechanical ventilator support in comparison of four $(33.3 \%)$ of non-deficient group. Eight (28.6\%) patients died in deficient group in comparison of one $(8.3 \%)$ who did not have ADAMTS-13 deficiency. However, mortality did not established statistically significant. Mean ADAMTS-13 level found low $0.35 \pm 0.3$ in sepsis patients in comparison of $0.89 \pm 0.41$ in healthy controls of same age and gender. Results are summarized in tableI, II \& III:

Among sepsis patientsof ADAMTS-13 deficient group, most frequent infection sites found were the urinary tract in $16(57.1 \%)$ followed by the respiratory system in 07 (25\%) patients. Escherichia coli was identified as the most common isolate in $12(42.9 \%)$ patients while Streptococcus

Table-I: General and haematological parameters in healthycontrols and sepsis patients.

\begin{tabular}{l|c|c}
\hline Characteristics & Healthy Controls $(\mathbf{n}=\mathbf{4 0})$ & Sepsis Patients $(\mathbf{n}=\mathbf{4 0 )}$ \\
\hline Mean Age \pm SD (Years) & $60 \pm 15$ & $61 \pm 15$ \\
\hline Gender $\mathrm{n}(\%)$ & - & - \\
\hline Male & $32(80 \%)$ & $32(80 \%)$ \\
\hline Female & $08(20 \%)$ & $08(20 \%)$ \\
\hline Mean \pm SD ADAMTS-13 Level $(\mathrm{IU} / \mathrm{ml})$ & $0.89 \pm 0.41$ & $0.35 \pm 0.3$ \\
\hline Mean \pm SD Leukocyte Count $\left(\times 10^{9} / \mathrm{l}\right)$ & $8.4 \pm 2.75$ & $20.2 \pm 6.87$ \\
\hline Mean \pm SD Platelet Count $\left(\times 10^{9} / 1\right)$ & $306 \pm 97.5$ & $20.4 \pm 5.8$ \\
\hline Mean \pm SD PT (Sec) & $15.4 \pm 1.2$ & $39.5 \pm 10.1$ \\
\hline Mean \pm SD aPTT $(\mathrm{Sec})$ & $32.8 \pm 2.2$ & \\
\hline
\end{tabular}

analysis of ADAMTS-13 levels by chromogenic ELISA assay, deficiency was found in $28(70 \%)$ patients while twelve (30\%) had normal antigen levels. Haematologic parameters were assessed and noted. Low ADAMTS-13 levels were found associated with low platelet counts. In 28 patients pneumonia in $03(10.7 \%)$, Staphylococcus aureus, Klebsiella, Candida, Neisseria meningitides in 02 (7.1\%) each and Bacillus fusiformis, Proteus, pseudomonas aeruginosa in 01 (3.6\%) each respectively. Two patients $(7.1 \%)$ with negative culture were detected Mycoplasma pneumonia on serolo- 
gy. More or less same frequency in site of infection and organisms was observed in non-deficient group. We found UTI is the frequent infection and E.coli is the leading cause of sepsis in our investigated adults. with sepsis did exhibit ADAMTS-13 deficiency which may have also contributed to sepsis induced thrombocytopenia. Many critical conditions like sepsis and TTP would require serial monitoring of ADAMTS-13 antigen levels and can be

Table-II: General and Haematological Characteristics in ADAMTS-13 Deficient and Non-deficient Groupof Sepsis Patients.

\begin{tabular}{l|c|c}
\hline Characteristics & $\begin{array}{c}\text { ADAMTS-13 Deficient } \\
\text { Group with Sepsis (n=28) }\end{array}$ & $\begin{array}{c}\text { ADAMTS-13 Non-deficient } \\
\text { Group with Sepsis (n=12) }\end{array}$ \\
\hline Mean Age \pm SD (Years) & $60( \pm 17)$ & $63.7( \pm 13)$ \\
\hline Gender n $(\%)$ & - & - \\
\hline Male & $22(78.6 \%)$ & $10(83.3 \%)$ \\
\hline Female & $06(21.4 \%)$ & $02(16.7 \%)$ \\
\hline Mean \pm SD ADAMTS-13 Level $(\mathrm{IU} / \mathrm{ml})$ & $0.19 \pm 0.08$ & $0.79 \pm 0.32$ \\
\hline Mean \pm SD Leukocyte Count $\left(\times 10^{9} / 1\right)$ & $20.3 \pm 7.75$ & $19.7 \pm 4.41$ \\
\hline Mean \pm SD Platelet Count $\left(\times 10^{9} / 1\right)$ & $97.1 \pm 71.0$ & $16.7 \pm 1.9$ \\
\hline Mean \pm SD PT (Sec) & $21.9 \pm 6.2$ & $31.6 \pm 7.2$ \\
\hline Mean \pm SD aPTT $(\mathrm{Sec})$ & $42.8 \pm 9.3$ & $\mathbf{2}$ \\
\hline
\end{tabular}

Table-III: Comparison of morbidity and mortality between ADAMTS-13 Deficient and Non-deficient sepsis patients.

\begin{tabular}{l|c|c|c}
\hline Characteristics & $\begin{array}{c}\text { ADAMTS-13 Deficient } \\
\text { Group with Sepsis (n=28) }\end{array}$ & $\begin{array}{c}\text { ADAMTS-13 Non-deficient } \\
\text { Group with Sepsis (n=12) }\end{array}$ & $\boldsymbol{p}$-value \\
\hline Mean \pm SD ICU Stay (Days) & $9.6(2.5)$ & $5.5(1.6)$ & $<0.01$ \\
\hline Mean \pm SD Hospital Stay (Days) & $19.2(7.6)$ & $10.2(3.6)$ & 0.012 \\
\hline Mechanical Ventilation n (\%) & $22(78.6 \%)$ & $04(33.3 \%)$ & 0.001 \\
\hline Mortality n (\%) & $08(28.6 \%)$ & $01(8.3 \%)$ & 0.344 \\
\hline
\end{tabular}

\section{DISCUSSION}

ADAMTS-13 geneis positioned on Chromosome nine (9q34). Primary ADAMTS-13 deficiency caused by defects in the ADAMTS-13 gene has been shown to cause thrombotic thrombocytopenic purpura (TTP). Secondary ADAMTS13 deficiency is seen in sepsis, acute inflammation, connective tissue diseases, and liver cirrhosis. The proposed mechanism of ADAMTS-13 deficiency in sepsis may includeits excessive utilization due to splitting of ultra large vWF by activated endothelium. Secondly, the state of inflammation, cytokines like interleukin-6 (IL-6) inhibitthe enzyme while simultaneously neutrophils release proteases which break down the ADAMTS-13.

Data is restricted in our country related to ADAMTS-13 deficiency in adult sepsis patients. A few studies have been carried out in the pediatric population. A large proportion of patients used as prognostic marker to prevent diseases related complications and mortality. Chromogenic ELISA based assay can provide accurate results with a rapid turnaround time.

In our study, the median age of the patients' suffered from sepsis was 65 years. This septic age group is close to a study conducted in France by Peigne et al who reported a median age of 59 years in their sepsis patients. However, 64\% patients in their study were males while in our $80 \%$ were males and $50 \%$ of the French sepsis patients found ADAMTS-13 deficient while in our investigated community, $70 \%$ patients were had ADAMTS-13 deficiency ${ }^{13}$. Another study conducted in the University Hospital Bern, Switzerland by Kremer Hovingaet alon 40 patients with sepsis. He compared sepsis patients with 40 healthy controls of the same age and gender and found ADAMTS-13 level significantly lower in sepsis patients than in healthy controls ${ }^{11}$. Claus et 
al has also demonstrated a significant correlation between sepsis and low ADAMTS-13 levels ${ }^{14}$.

In our study population, patients with low ADAMTS-13 levels had decline in platelet counts, these findings are in accordance to the results of studies conducted in Paris by Peigne et al13 and Clemens et al ${ }^{15}$ who also reported thrombocytopenia in their sepsis patients. This might be due to low levels of ADAMTS-13, increase ultralargev WF secondary to infection and host inflammation.

Our results illustrated the correlation between fall in ADAMTS-13 level, the length of ICU /hospital stay and use of mechanical ventilation. However, there was a no statistically significant association between low ADAMTS 13 level and mortality of the patients $(p=0.34)$. This finding is contrary to Azfar et al who reported decrease ADAMTS-13 a significant risk factor for mortality in the Saudi population ${ }^{16}$. Martin et al observed ADAMTS-13 level are diminished in patients with severe sepsis in analogy ofhealthy subjects and who had developed organ failure unrelated to sepsis ${ }^{17}$. However, their study also has showed no clear association between ADAMTS-13 levels and mortality while Peigne et al ${ }^{13}$ and Athira et al 25 have demonstrated a significantly higher mortality rate with ADAMTS-13 deficiency in sepsis. Sarig et al ${ }^{18}$ and Masias et al ${ }^{19}$ proposed ADAMTS-13 levela decisive tool in management of micro angiopathies. Smeets et al reported decrease ADAMTS-13 level in a Caucasian patient suffered from sepsis secondary to Gram-negative bacillus Capnocytophaga canimorsus ${ }^{20}$. Thrombotic microangiopathy (TMA) in sepsis may be facilitated by ultralargev WF multimers whose level could be increased because of (secon-dary) ADAMTS-13 deficiency ${ }^{21}$. These secondary thrombotic microangiopathies like in TTP, DIC and sepsis possibly could benefit from ADAMTS-13 replacement or a vWF-targeting therapy ${ }^{12}$. Matevosyan et al emphasizes on thorough investigations including ADAMTS-13 levels in TMA patients to differentiate the underlying mechanism $^{22}$. In a group of cohort Li et al described ADAMTS-13 level is not an independent predic- tor of poor outcomes in sepsis but instead appears to be a marker of disease acuity ${ }^{23}$. Chen et al elaborate dultra large vWF due to lesser ADAMTS-13 levels in inflammation are chiefly responsible for thrombosis and end organ damage in sepsis ${ }^{24}$.

We tried to explore the role of ADAMTS-13 in adult sepsis patients and to evaluate its relationship to disease severity and outcome. The limitation in our study was that, ADAMTS-13 level only assayed on day three of hospital admission, after laboratory confirmation of microbiological infection and serial monitoring of ADAMTS-13 level in subsequent days was not done due to limited project budget.

\section{Author's Contribution}

Dr Owais Ali composed information, analyzed data and wrote the initial manuscript. Dr Chaudhry Altaf Hussain perceived the design, critically figure out the article and provided guidance. Dr Dawood oversight and monitored all operative activity. Dr Rafia Mahmood did the literature review and helped in manuscript preparation. Dr Syeda Sarwat Fatima and Dr Taqdees Fatima were the primary physicians who helped in recruitment of patients and performed bench work. All authors read and approved the final manuscript. Research officer, Mr. Bilal Rao helped with the statistical analysis.

\section{CONCLUSION}

ADAMTS-13 is an important prognostic marker in patients hospitalized due to sepsis. Majority of sepsis patients have low ADAMTS13 levels compared to healthy subjects. Lower ADAMTS-13 levels are associated with increase in hospital stay and sepsis induced thrombocytopenia. However, further studies with larger number of patients and long term follow-up is required to evaluate the role of ADAMTS-13 deficiency in sepsis patients.

\section{CONFLICT OF INTEREST}

The authors declare that they have no conflict of interests. 


\section{REFERENCES}

1. Singer M, Deutschman CS, Seymour CW, Shankar-Hari M, Annane D, Bauer M, et al. The third International consensus definitions for sepsis and septic shock (sepsis-3). J Am Med Assoc 2016; 315(8): 801-10.

2. Murphy SL, Xu J, Kochanek KD. Deaths: final data for 2010. Natl Vital Stat Rep 2013; 61(4): 1-117.

3. National Institute of General Medical Sciences. Sepsis Fact Sheet. 2014.

4. Lenting PJ, Casari C, Christophe OD, et al. von Willebrand factor: the old, the new and the unknown. J Thromb Haem 2012; 10(12): 2428-37.

5. Stockschaelder M, Schneppenheim R, Budde U. Update on von Willebrand factor multimers: focus on high-molecular-weightmultimers and their role in hemostasis. Blood Coagul Fibrin 2014; 25(1); 206-16.

6. American College of Chest Physicians/Society of Critical Care Medicine Consensus Conference: definitions for sepsis and organ failure and guidelines for the use of innovative therapies in sepsis. Crit Care Med 1992; 20(6): 864-74.

7. TTP and ADAMTS13: When Is Testing Appropriate? Mannucci PM, Peyvandi F. Hematology Am Soc Hematol Educ Program 2007: 121-6.

8. Xing K, Murthy S, Liles WC, Singh JM. Clinical utility of biomarkers of endothelial activation in sepsis a systematic review. Crit Care 2012; 16(1): r7.

9. Aibar J, Castro P, Espinosa G, Fernández S, ernández C, Rinaudo M, et al. ADAMTS-13 in Critically Ill Patients with Septic Syndromes and Non infectious systemic Inflammatory Response Syndrome. Shock 2015; (6): 556-62.

10. Lin JJ, Chan OW, Hsiao HJ. Decreased ADAMTS-13 Activity is associated with disease severity and outcome in pediatric severe sepsis. Medicine (Baltimore) 2016; 95(16): e3374.

11. Hovinga JAK, Zeerleder $S$, Kessler $P$, Romani de Wit $T$, van Mourik JA, Hack CE, ten Cate H, et al. ADAMTS-13, von Wille brand factor and related parameters in severe sepsis and septic shock. J Thromb Haemost 2007; 5(11): 2284-90.

12. Schwameis $M$, Schörgenhofer $C$, Assinger A, Steiner MM, Jilma B. Von Willebrand factor excess and ADAMTS-13 deficiency: a unifying pathomechanism linking inflammation to thrombosis in DIC, malaria, and TTP. Thromb Haemost 2015; 113(4): 708-18.

13. Peigne V, Azoulay E, Coquet I, Mariotte E, Darmon M, Legendre
$\mathrm{P}$, et al. The prognostic value of ADAMTS-13 (adisintegrin and metalloprotease with thrombospondin type 1 repeats, member 13) deficiency in septic shock patients involves interleukin-6 and is not dependent on disseminated intravascular coagulation. Crit Care 2013; 17(6): R273.

14. Claus RA, Bockmeyer CL, Budde U, Kentouche K, Sossdorf M, Hilberg $\mathrm{T}$, et al. Variation in the ratio between vWF factor andits cleaving protease during systemic inflammation and association with severity. Thromb Haemost 2009; 101(2): 239-47.

15. Bockmeyer CL, Claus RA, Budde U, Kentouche K, et al. Inflammation Associated ADAMTS-13 Deficiency promotes Formation of Ultra-Large Von Willebr and Factor. Haematologica January 2008; 93: 137-40.

16. Azfar MF, Khan MF, Habib SS, Al Aseri Z, Zubaidi AM, Aguila DO, et al. Prognostic value of ADAMTS-13 deficiency in patients with severe sepsis and septic shock. Clin Invest Med 2017; 40(2): e49-58.

17. Martin K, Borgel D, Lerolle N, Feys HB, Trinquart L, Vanhoorelbeke K, et al. Decreased ADAMTS-13 (Adisintegrinlike and metalloprotease with thrombospondin type 1 repeats) is associated with a poor prognosis in sepsis-inducedorgan failure. Crit Care Med 2007; 35(10): 2375-82.

18. Sarig G. ADAMTS-13 in the diagnosis and management of thrombotic microangiopathies. Rambam Maimonides Med J 2014; 5(4): 1-5.

19. Masias C, Cataland SR. The role of ADAMTS13 testing in the diagnosis and management of thrombotic microangiopathies and thrombosis. Blood 2018; 132(9): 903-10.

20. Secondary thrombotic microangiopathy with severely reduced ADAMTS-13 activity in a patient with Capnocytophaga canimorsus sepsis: A case report 2018; 58(10): 2426-29.

21. Levi $M$, Scully $M$, Singer $M$. The role of ADAMTS-13 in the coagulopathy of sepsis. J Thromb Haemost 2018; 16(4): 646-51.

22. Matevosyan K, Sarode R. Thrombosis, Microangiopathies, and Inflammation. Semin Thromb Hemost 2015; 41(6): 556-62.

23. Clinical features and outcomes in patients with thrombotic microangiopathy not associated with severe ADAMTS13 deficiency. Transfusion 2017; 57(9): 2151-58.

24. Chen J, Chung DW. Inflammation, von Willebrand factor and ADAMTS-13. Blood 2018: blood-2018-02-769000.

25. Unnikrishnan A, Doonan B, Ali A, Delaune JD, Jester G, Wang $Y$, et al. Significance of ADAMTS-13 activity level in severe sepsis and septic shock. Blood 2017; 130(1): 4837. 